\title{
FEEDBACK LINEARIZATION OF AN ACTIVE MAGNETIC BEARING SYSTEM OPERATED WITH A ZERO-BIAS FLUX
}

\author{
ARKADIUSZ MYSTKOWSKI ${ }^{a}, \operatorname{VADIM~KAPARIN~}^{b}$, ÜLLE KOTTA $^{b}$, EWA PAWLUSZEWICZ $^{a, *}$, \\ MARIS TÕNSO ${ }^{b}$
}

\author{
${ }^{a}$ Department of Automatic Control and Robotics \\ Bialystok University of Technology, ul. Wiejska 45A, 15-351 Bialystok, Poland \\ e-mail: \{a.mystkowski, e.pawluszewicz\}@pb.edu.pl \\ ${ }^{b}$ Department of Software Science \\ Tallinn University of Technology, Akadeemia tee 21, 12618 Tallinn, Estonia \\ e-mail: \{vkaparin, kotta, maris\} @cc.ioc.ee
}

\begin{abstract}
Input-output linearization by state feedback is applied to a flux-controlled active magnetic bearing (AMB) system, operated in the zero-bias mode. Two models of the AMB system are employed. The first one is described by the third-order dynamics with a flux-dependent voltage switching scheme, whereas the second one is the fourth-order system, called selfsensing $\mathrm{AMB}$, since it does not require the measurement of the rotor position. In the case of that system we had to find the flat outputs to guarantee its stability. The proposed control schemes are verified by means of numerical simulations performed within the Matlab environment.
\end{abstract}

Keywords: active magnetic bearing, flux control, zero-bias, feedback linearization, flat outputs.

\section{Introduction}

Feedback linearization is a well-known and conceptually simple set of techniques for control systems with smooth nonlinearities. The essence of the approach is to compensate the nonlinearities and make the system, in the closed-loop, behave as a linear one. There exist various problem statements which are applicable to different system classes. The linearity is achieved either for (extended) state equations or only for input-output (i/o) equations. The feedback is looked for in the class of state or output (measurement) feedbacks; in both cases static and dynamic feedbacks are sought. Moreover, the system itself may be described either by state or i/o equations. It has to be noticed that not all nonlinear i/o equations can be transformed into state equations. In addition to these mainstream approaches, there exist numerous different problem statements, including approximate linearization.

Note that the possibility to linearize all state equations depends on restrictive integrability conditions and as such the approach is applicable only to a limited

*Corresponding author subclass of nonlinear systems (Isidori, 1995; Conte et al., 2007; Nijmeijer and van der Schaft, 1990). For i/o linearizability the solvability conditions are milder; the only requirements are right invertibility and stability of zero dynamics (Nijmeijer and van der Schaft, 1990).

In case the zero dynamics are unstable for original system outputs, it is possible to look for another set of 'virtual' outputs with respect to which the system has stable zero dynamics. The related approach is to search for the so-called flat outputs that will result in a system without zero dynamics (Lévine, 2009; Sira-Ramírez and Agrawal, 2004). In general, the problem of finding flat outputs is not an easy task and still lacks a good constructive general solution. Nevertheless, for the special case we will face in this paper-the static state feedback linearizable system - there exists a simple method to find flat outputs. Once these are found, there is no need to use the exact linearization technique with complicated state transformation, but it is enough to apply the i/o linearization method to these 'virtual' outputs.

AMB models, linearized around an operating point, are sensitive to perturbations and work properly only in 
non-zero bias mode (see, e.g., Lindlau and Knospe, 2002; Chen and Knospe, 2005; Jastrzebski et al., 2014). There exist many papers that apply feedback linearization to make the system equations exactly linear; see a brief overview below. The paper by Joo and Seo (1997) shows that the third-order electromagnetic suspension system is input-state feedback linearizable and the nonlinear feedback controller is robust against matching constant input disturbances and mass perturbations. Naz et al. (2013) applied input-state linearization for a simpler second-order magnetic levitation system. State-space linearization was also applied to a perturbed magnetic suspension system by Mittal and Menq (1997). Robustness to unmatched uncertainties in the linearized system is achieved via the development of additional compensation algorithms. In the work by Charara et al. (1996) the controller based on input-output linearization for a magnetic levitation system without premagnetization was designed to stabilize an inertial wheel (without premagnetization). Note that here the zero dynamics were proven to be stable earlier. Matsumura et al. (1999) applied the input-state linearization to the AMB system controlled by deviations from a steady-state voltage. The input-state linearization approach was also applied to a magnetic bearing system with two voltage inputs by Baloh et al. (2000). In the work of Ghosh et al. (2000) the i/o lineariztion was applied to an AMB system using a variable bias voltage to avoid singularity in the feedback, typically faced in the zero-bias mode operation. Finally, the only paper on differential flatness of an AMB system, to the best of the authors' knowledge, is that by Lévine et al. (1996), which addressed both the current and voltage control cases.

No papers among these references address feedback linearization of a flux-controlled AMB system in zerobias mode. Therefore, we discuss this problem in our paper. Unlike the current-voltage control, the flux control is more direct and has the advantage that the attractive force is independent of the rotor position. This means that the whole AMB control system can be divided into the inner flux-controlled nonlinear part and the outer part, where the rotor position is controlled by a linear controller. This decoupling allows simplifications in the control design. Moreover, the application of the flux-based control decreases the force estimation error, and the controller can achieve a larger bandwidth than a classical current-controlled AMB system. For a detailed comparison between flux- and current-control of the AMB system, with experimental verification, we refer the reader to Jastrzebski et al. (2014).

The goal of this paper is to apply the i/o linearization technique to two AMB systems. The first one is a flux-controlled AMB system operated with zero-bias. The system is described by third-order nonlinear dynamics. The zero-bias control strategy is applied using a switching voltage sequence. The switching scheme allows us to minimize the control fluxes $\phi_{1}$ and $\phi_{2}$ since at every time instant at least one of the electromagnets is inactive. Thus this system minimizes energy losses. The second system is the fourth-order system which has two voltages $u_{1}, u_{2}$ as control inputs and two currents $i_{1}, i_{2}$ as the outputs. Since this system is not using rotor position measurements it is called a self-sensing AMB system. When applying the feedback linearization technique to the third-order model, we face some issues that are not covered by theory. First, the AMB model is not described completely by smooth nonlinearities. Second, in the operating region, the system frequently passes through singular points, where the generalized electromagnetic flux is zero. Third, one has to deal with actuator constraints. In the case of the fourth-order AMB system with two voltages control, we develop, based on a system flatness property, a flux-dependent voltage switching scheme, depending on the electromagnet whose flux is different from zero.

The paper is organized as follows. Section 2 recalls the solution of the i/o linearization problem by state feedback. In Section 3.1 the linearizing feedback is constructed for the third-order AMB system, whereas in Section 3.2 the fourth-order system is linearized using the feedback that switches between two controls, depending on the electromagnet whose flux is different from zero. The proposed control schemes are verified based on simulations in Section 4, whereas Section 5 draws concluding remarks.

\section{I/O linearization by state feedback: A brief overview}

In the i/o linearization approach, the objective is to linearize the map between the new input $v$ and the output $y$. Below we recall the solution by Isidori (1995). The AMB systems studied in this paper are of the form

$$
\begin{gathered}
\dot{x}=f(x)+\sum_{j=1}^{m} g_{j}(x) u_{j}, \\
y_{i}=h_{i}(x), \quad i=1, \ldots, m,
\end{gathered}
$$

where $x \in \mathbb{R}^{n}, u=\left[\begin{array}{lll}u_{1} & \cdots & u_{m}\end{array}\right]^{\mathrm{T}} \in \mathbb{R}^{m}, y=$ $\left[\begin{array}{lll}y_{1} & \cdots & y_{m}\end{array}\right]^{\mathrm{T}} \in \mathbb{R}^{m}$ and $m<n$.

Definition 1. Given a system of the form (1), one can state that the $i$-th output $y_{i}$ has relative degree $r_{i}$ if

$$
\begin{aligned}
L_{g_{j}} L_{f}^{k} h_{i}(x)= & 0, \\
& j=1, \ldots, m ; \quad k=0, \ldots, r_{i}-2
\end{aligned}
$$

and the row vector

$$
\left[\begin{array}{lll}
L_{g_{1}} L_{f}^{r_{i}-1} h_{i}(x) & \cdots & L_{g_{m}} L_{f}^{r_{i}-1} h_{i}(x)
\end{array}\right]
$$

is nonzero. 
Here $L_{f}$ is the Lie derivative, $L_{f} h_{i}(x):=$ $\left(\partial h_{i}(x) / \partial x\right) f$. Observe that $r_{i}$ is the lowest-order timederivative of $y_{i}$ that explicitly depends on control $u$. Assume that each output $y_{i}$ possesses a finite relative degree and construct the so-called decoupling matrix

$$
\begin{aligned}
& \mathbf{A}(x) \\
& :=\left[\begin{array}{ccc}
L_{g_{1}} L_{f}^{r_{1}-1} h_{1}(x) & \cdots & L_{g_{m}} L_{f}^{r_{1}-1} h_{1}(x) \\
\vdots & & \vdots \\
L_{g_{1}} L_{f}^{r_{m}-1} h_{m}(x) & \cdots & L_{g_{m}} L_{f}^{r_{m}-1} h_{m}(x)
\end{array}\right] .
\end{aligned}
$$

Definition 2. A system of the form (1) has a vector relative degree $\left[\begin{array}{lll}r_{1} & \cdots & r_{m}\end{array}\right]$ if the matrix $\mathbf{A}$ has full rank.

Definition 3. A system of the form (1) is called (strictly) $i / o$ linearizable if there exists a regular static state feedback of the form $u=\alpha(x)+\beta(x) v$ with a new input vector $v=\left[\begin{array}{lll}v_{1} & \cdots & v_{m}\end{array}\right]^{\mathrm{T}}$ and nonsingular $\beta(x)$ such that, in the closed-loop,

$$
y_{i}^{\left(r_{i}\right)}=v_{i}, \quad i=1, \ldots, m .
$$

For a system (1) with a vector relative degree the i/o linearization problem is always solvable and the required control is

$$
u=\mathbf{A}^{-1}(x)\left[\begin{array}{c}
v_{1}-L_{f}^{r_{1}} h_{1}(x) \\
\vdots \\
v_{m}-L_{f}^{r_{m}} h_{m}(x)
\end{array}\right] .
$$

Note that the system (5) is, in addition, decoupled; the decoupling is achieved as a by-product of i/o linearization. Though for i/o linearizability the solvability conditions are mild, the approach is not useful when the zero dynamics of the system are unstable. A difficulty in unstable zero dynamics is that some state variables will be unbounded, and, as a consequence, also the control will be unbounded. In such a case one possibility is to look for a fictitious (virtual) output with respect to which the system has stable zero dynamics or for a flat output that results in a closed-loop system without zero dynamics.

Finally, a linear controller can now be designed for the linear model (5). To stabilize the linear system, the new input $v_{i}$ may be taken as

$$
v_{i}=\left(y_{d}^{i}\right)^{\left(r_{i}\right)}-\boldsymbol{\alpha}_{i}^{\mathrm{T}} \mathbf{e}_{i},
$$

where $y_{d}^{i}$ stands for a desired output,

$$
\begin{aligned}
\boldsymbol{\alpha}_{i}^{\mathrm{T}} & :=\left[\begin{array}{llll}
\alpha_{i}^{0} & \alpha_{i}^{1} & \cdots & \alpha_{i}^{r_{i}-1}
\end{array}\right], \\
\mathbf{e}_{i} & :=\left[\begin{array}{llll}
e_{i} & \dot{e}_{i} & \cdots & e_{i}^{\left(r_{i}-1\right)}
\end{array}\right]^{\mathrm{T}}
\end{aligned}
$$

with $e_{i}:=y_{i}-y_{d}^{i}$. Stabilization is guaranteed if the real numbers $\alpha_{i}^{j}$ are chosen such that the polynomial $s^{r_{i}}+$ $\alpha_{i}^{r_{i}-1} s^{r_{i}-1}+\cdots+\alpha_{i}^{1} s+\alpha_{i}^{0}$ is Hurwitz.

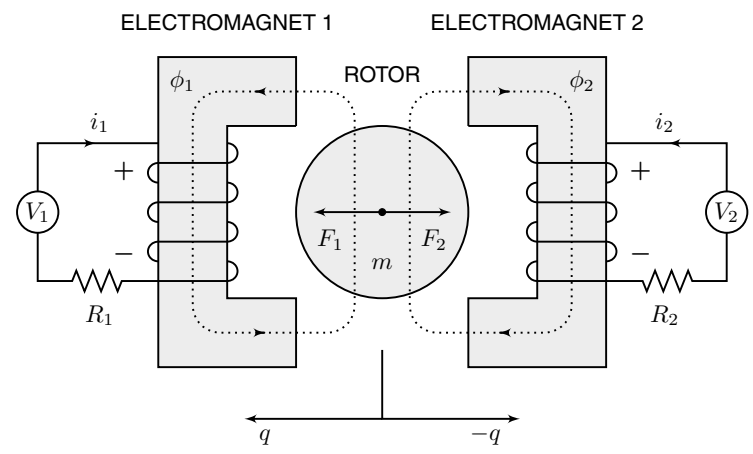

Fig. 1. Scheme of 1-DOF AMB.

\section{AMB system}

Consider a simplified one-degree-of-freedom (1-DOF) AMB model, which consists of two opposite and presumably identical electromagnetic actuators (electromagnets) with resistances $R_{1}, R_{2}$ and currents $i_{1}, i_{2}$, respectively (see Fig. 1). These electromagnets generate fluxes $\phi_{1}, \phi_{2}$ and further the attractive forces $F_{1}$, $F_{2}$ acting on the rotor with the mass $m$. In order to control the position $q$ of the rotor to the stable point $q=0$, the voltage inputs of the electromagnets $V_{1}$ and $V_{2}$ are used. Moreover, denote by $\mu_{0}, A_{g}$, and $N$ the permeability of free space $\left(=1.25 \times 10^{-6} \mathrm{H} / \mathrm{m}\right)$, the cross sectional area of the air gap, and the number of turns of the coil of each electromagnet, respectively.

In order to simplify the presentation, the 1-DOF model of the AMB system is considered. However, this simplification is reasonable, since the full (5-DOF) AMB system can be i/o decoupled into 1-DOF sub-models if the rotor is rigid. Many AMB rotor applications operate below the first bending natural frequency of the rotor, thus assuming the rotor to be a rigid body. Also some nonlinear effects negligible for the AMB system are omitted, such as eddy-currents (iron loss), hysteresis loss, ohmic effects, dynamic induction loss, resistance loss and losses caused by dynamic field changes. Note that most of these losses are reduced or eliminated by the zero bias control which is applied with the switching strategy (9).

3.1. Third-order system. The dynamics of the simplified 1-DOF AMB model with a zero-bias flux may be described by means of the following nonlinear state equations:

$$
\begin{aligned}
\dot{x}_{1} & =x_{2}, \\
\dot{x}_{2} & =\frac{x_{3}\left|x_{3}\right|}{\kappa}, \\
\dot{x}_{3} & =\frac{u}{N}, \\
y & =x_{1},
\end{aligned}
$$


where $x_{1}$ and $x_{2}$ denote the position $q[\mathrm{~m}]$ and velocity $\dot{q}$ $[\mathrm{m} / \mathrm{s}]$ of the rotor mass, respectively, $x_{3}$ is the generalized electromagnetic flux $\phi:=\phi_{1}-\phi_{2}[\mathrm{~Wb}]$, and $\kappa:=$ $m \mu_{0} A_{g}$. The control input $u$ is the generalized voltage [V] under the following flux-dependent voltage switching scheme:

$$
\begin{array}{lll}
V_{1}=u, & V_{2}=0 & \text { when } \phi \geq 0, \\
V_{2}=-u, & V_{1}=0 & \text { when } \phi<0 .
\end{array}
$$

3.1.1. Input-output linearization. To apply the control (6), from (8) compute

$$
\begin{aligned}
& \dot{y}=x_{2}, \\
& \ddot{y}=\frac{x_{3}\left|x_{3}\right|}{\kappa} .
\end{aligned}
$$

Note that the absolute value function $\left|x_{3}\right|$ is not differentiable at $x_{3}=0$; however, except this point we have $\partial\left|x_{3}\right| / \partial t=\operatorname{sgn}\left(x_{3}\right) \dot{x}_{3}$. Therefore,

$$
\dddot{y}=\frac{u\left(\left|x_{3}\right|+\operatorname{sgn}\left(x_{3}\right) x_{3}\right)}{N \kappa} .
$$

Note first that the relative degree $r$ of $y$ is 3. From (8), $f=\left[\begin{array}{lll}x_{2} & \left|x_{3}\right| x_{3} / \kappa & 0\end{array}\right]^{\mathrm{T}}, g:=g_{1}=\left[\begin{array}{lll}0 & 0 & 1 / N\end{array}\right]^{\mathrm{T}}$ and $h:=h_{1}=x_{1}$; thus $L_{f} h=x_{2}, L_{f}^{2} h=\left|x_{3}\right| x_{3} / \kappa$ and the decoupling matrix is

$$
\mathbf{A}(x)=\left[L_{g} L_{f}^{2} h\right]=\left[\frac{1}{N \kappa}\left(\left|x_{3}\right|+\operatorname{sgn}\left(x_{3}\right) x_{3}\right)\right] .
$$

Since $L_{f}^{3} h=0$, from (6) we get

$$
u=\frac{v N \kappa}{\left|x_{3}\right|+\operatorname{sgn}\left(x_{3}\right) x_{3}} .
$$

In (13) one may choose $v=y_{d}^{(3)}-\boldsymbol{\alpha}^{\mathrm{T}} \mathbf{e}$, where $y_{d}$ is a desired output, $\mathbf{e}=\left[\begin{array}{lll}e & \dot{e} & \ddot{e}\end{array}\right]^{\mathrm{T}}, e=y-y_{d}$, and $\boldsymbol{\alpha}^{\mathrm{T}}=$ $\left[\begin{array}{ccc}\alpha^{0} & \alpha^{1} & \alpha^{2}\end{array}\right]$ satisfies the condition that the polynomial $s^{3}+\alpha^{2} s^{2}+\alpha^{1} s+\alpha^{0}$ is Hurwitz.

Observe that in the control law (13) we have a singularity at $x_{3}=0$. We may modify the control law (13) as

$$
u= \begin{cases}\frac{v N \kappa}{\left|x_{3}\right|+\operatorname{sgn}\left(x_{3}\right) x_{3}} & \text { if }\left\|x_{3}\right\|>\epsilon, \\ -k \operatorname{sgn}\left(x_{3}\right) & \text { if }\left\|x_{3}\right\| \leq \epsilon,\end{cases}
$$

where $\epsilon$ is a design parameter of the controller that specifies the boundary of the magnetic flux near the origin and $k$ is a constant feedback gain. Let the desired trajectory $y_{d}=0$; then $e=y, \dot{e}=\dot{y}, \ddot{e}=\ddot{y}$ and $v=$ $-\left(\alpha^{2} \ddot{y}+\alpha^{1} \dot{y}+\alpha^{0} y\right)$. Plugging this into the expression for $u$ gives the new control stabilizing the system,

$$
u= \begin{cases}\frac{N \kappa\left(\alpha^{2} \ddot{y}+\alpha^{1} \dot{y}+\alpha^{0} y\right)}{\left|x_{3}\right|+\operatorname{sgn}\left(x_{3}\right) x_{3}} & \text { if }\left\|x_{3}\right\|>\epsilon, \\ -k \operatorname{sgn}\left(x_{3}\right) & \text { if }\left\|x_{3}\right\| \leq \epsilon .\end{cases}
$$

3.2. Fourth-order system. The mathematical model of the simplified self-sensing AMB model may be described by the state equations (Maslen, 2013)

$$
\begin{aligned}
& \dot{x}_{1}=x_{2}, \\
& \dot{x}_{2}=\frac{x_{3}^{2}-x_{4}^{2}}{A_{g} m \mu_{0}}+\frac{f_{e}}{m}, \\
& \dot{x}_{3}=\frac{1}{N}\left(u_{1}-R \frac{2\left(s_{0}-x_{1}\right)}{A_{g} N \mu_{0}} x_{3}\right), \\
& \dot{x}_{4}=\frac{1}{N}\left(u_{2}-R \frac{2\left(s_{0}+x_{1}\right)}{A_{g} N \mu_{0}} x_{4}\right), \\
& y_{1}=\frac{2\left(s_{0}-x_{1}\right)}{A_{g} N \mu_{0}} x_{3}, \\
& y_{2}=\frac{2\left(s_{0}+x_{1}\right)}{A_{g} N \mu_{0}} x_{4},
\end{aligned}
$$

where the states $x_{1}, x_{2}, x_{3}$, and $x_{4}$ denote the position $q[\mathrm{~m}]$, velocity $\dot{q}[\mathrm{~m} / \mathrm{s}]$, and the fluxes $\phi_{1}$ and $\phi_{2}$, respectively. In order to control the position $q$ of the rotor mass to the stable point $q=0$, the voltage inputs of the electromagnets $u_{1}:=V_{1}$ and $u_{2}:=V_{2}$ are used. The currents $i_{1}$ and $i_{2}$ are used as the outputs $y_{1}$ and $y_{2}$, respectively. Moreover, $R=R_{1}=R_{2}$ is the resistance of both the coils, $f_{e}$ is an external disturbance force and $s_{0}$ denotes the length of the air gap. Note that the variables $x_{3}$ and $x_{4}$ are not measurable. However, if one can measure the position $x_{1}$, then $x_{3}$ and $x_{4}$ can be computed from the outputs $y_{1}$ and $y_{2}$.

3.2.1. Input-output linearization. Again, according to the theory described in Section 2, we first find the relative degrees of the system outputs. For that purpose, compute

$$
\begin{aligned}
\dot{y}_{1}= & \frac{2\left(s_{0}-x_{1}\right) u_{1}}{A_{g} N^{2} \mu_{0}} \\
& -\frac{4 R\left(s_{0}-x_{1}\right)^{2} x_{3}}{A_{g}^{2} N^{3} \mu_{0}^{2}}-\frac{2 x_{2} x_{3}}{A_{g} N \mu_{0}}, \\
\dot{y}_{2}= & \frac{2\left(s_{0}+x_{1}\right) u_{2}}{A_{g} N^{2} \mu_{0}} \\
& -\frac{4 R\left(s_{0}+x_{1}\right)^{2} x_{4}}{A_{g}^{2} N^{3} \mu_{0}^{2}}+\frac{2 x_{2} x_{4}}{A_{g} N \mu_{0}} .
\end{aligned}
$$

Both $\dot{y}_{1}$ and $\dot{y}_{2}$ already depend on input. Thus the relative degrees of the system are $r_{1}=r_{2}=1$. Next, using (4), compute the decoupling matrix

$$
\mathbf{A}(x)=\frac{\partial \dot{y}}{\partial u}=\left[\begin{array}{cc}
\frac{2\left(s_{0}-x_{1}\right)}{A_{g} N^{2} \mu_{0}} & 0 \\
0 & \frac{2\left(s_{0}+x_{1}\right)}{A_{g} N^{2} \mu_{0}}
\end{array}\right] .
$$

Obviously, $\operatorname{rank}(\mathbf{A}(x))=2$ everywhere owing to the condition $s_{0}>x_{1}$. Note that from the physics of the 
AMB system it follows that, if $x_{1}=\left|s_{0}\right|$, then the AMB system is on the boundary limit.

To find the linearizing control, we first, by (6), compute

$$
\begin{gathered}
\mathbf{A}^{-1}(x)=\left[\begin{array}{cc}
\frac{A_{g} N^{2} \mu_{0}}{2\left(s_{0}-x_{1}\right)} & 0 \\
0 & \frac{A_{g} N^{2} \mu_{0}}{2\left(s_{0}+x_{1}\right)}
\end{array}\right], \\
L_{f} h=\left[\begin{array}{c}
2 x_{3} \frac{-2 R\left(s_{0}-x_{1}\right)^{2}-A_{g} N^{2} \mu_{0} x_{2}}{A_{g}^{2} N^{3} \mu_{0}^{2}} \\
2 x_{4} \frac{-2 R\left(s_{0}+x_{1}\right)^{2}+A_{g} N^{2} \mu_{0} x_{2}}{A_{g}^{2} N^{3} \mu_{0}^{2}}
\end{array}\right]
\end{gathered}
$$

and then

$$
\begin{aligned}
& u_{1}=\frac{2 R x_{3}\left(s_{0}-x_{1}\right)}{A_{g} N \mu_{0}}+\frac{N\left(A_{g} N \mu_{0} v_{1}+2 x_{2} x_{3}\right)}{2\left(s_{0}-x_{1}\right)}, \\
& u_{2}=\frac{2 R x_{4}\left(s_{0}+x_{1}\right)}{A_{g} N \mu_{0}}+\frac{N\left(A_{g} N \mu_{0} v_{2}-2 x_{2} x_{4}\right)}{2\left(s_{0}+x_{1}\right)},
\end{aligned}
$$

yielding $\dot{y}_{1}=v_{1}$ and $\dot{y}_{2}=v_{2}$. Unfortunately, now, unlike for the model (8), $r_{1}+r_{2}<n$. The closed-loop system equations can be transformed into the normal form via a state diffeomorphism, if we define the new state variables as

$$
\begin{aligned}
& z_{1}:=y_{1}=\frac{2\left(s_{0}-x_{1}\right)}{A_{g} N \mu_{0}} x_{3}, \quad z_{3}:=x_{1}, \\
& z_{2}:=y_{2}=\frac{2\left(s_{0}+x_{1}\right)}{A_{g} N \mu_{0}} x_{4}, \quad z_{4}:=x_{2} .
\end{aligned}
$$

By means of $\mathrm{i} / \mathrm{o}$ linearization, the dynamics of the closed-loop system in the transformed coordinates are decomposed into a linear part of order $r_{1}+r_{2}=2$,

$$
\begin{aligned}
& \dot{z}_{1}=v_{1}, \\
& \dot{z}_{2}=v_{2}, \\
& y_{1}=z_{1}, \\
& y_{2}=z_{2},
\end{aligned}
$$

and the (unobservable) non-linear part of order $n-r_{1}-$ $r_{2}=2$,

$$
\begin{aligned}
& \dot{z}_{3}=z_{4} \\
& \dot{z}_{4}=\frac{f_{e}}{m}+\frac{A_{g} N^{2} \mu_{0} z_{1}^{2}}{4 m\left(s_{0}-z_{3}\right)^{2}}-\frac{A_{g} N^{2} \mu_{0} z_{2}^{2}}{4 m\left(s_{0}+z_{3}\right)^{2}} .
\end{aligned}
$$

The key question is whether the states of the internal dynamics (23) will remain bounded. The stability of the internal dynamics can be studied through the zero dynamics. Note that the internal dynamics (23) depend on the output variables via $z_{1}$ and $z_{2}$. The aim is to stabilize the linear part (22) using controller (7) and see what will happen with the nonlinear part (23). Since $r_{1}=r_{2}=1$, the controller has the form $v_{1}=-\alpha_{1}^{0} y_{1}, v_{2}=-\alpha_{2}^{0} y_{2}$, where $\alpha_{1}^{0}>0, \alpha_{2}^{0}>0$. Now the linear part (22) is stable, i.e., $y_{1} \rightarrow 0, y_{2} \rightarrow 0$, if $t \rightarrow \infty$. This also means that the state variables $z_{1}$ and $z_{2}$ converge to zero, and therefore (23) becomes

$$
\begin{aligned}
& \dot{z}_{3}=z_{4}, \\
& \dot{z}_{4}=\frac{f_{e}}{m}
\end{aligned}
$$

as $t \rightarrow \infty$. Equation (24), known as the zero dynamics, is obviously unstable.

3.2.2. Flat outputs. The objective of this subsection is to derive artificial outputs $\tilde{y}_{1}$ and $\tilde{y}_{2}$ that yield a feedback linearized model with the dimension $n=r_{1}+r_{2}$. To determine the flat outputs, for the system (16a) we first compute the sequence of subspaces of differential one-forms, defined by the following recursive formula:

$$
\begin{aligned}
\mathcal{H}_{1} & :=\operatorname{span}\{\mathrm{d} x\}, \\
\mathcal{H}_{k+1} & :=\left\{\omega \in \mathcal{H}_{k} \mid \dot{\omega} \in \mathcal{H}_{k}\right\}, \quad k \geq 1,
\end{aligned}
$$

(see more in the work Conte et al. (2007)). These subspaces determine the so-called Brunovsky form. Observe that the last non-zero subspace is

$$
\mathcal{H}_{3}=\operatorname{span}\left\{\mathrm{d} x_{1}\right\}
$$

The subspace $\mathcal{H}_{3}$, i.e., $\mathrm{d} x_{1}$, defines the starting element of the first Brunovsky chain. Second, consider $\mathcal{H}_{2}$ and observe that this subspace does not define the new Brunovsky chain, since

$$
\mathcal{H}_{2}=\operatorname{span}\left\{\mathrm{d} x_{1}, \mathrm{~d} x_{2}\right\}=\operatorname{span}\left\{\mathrm{d} x_{1}, \mathrm{~d} \dot{x}_{1}\right\} .
$$

However, for our system 16a we have that

$$
\mathcal{H}_{1}=\left\{\mathrm{d} x_{1}, \mathrm{~d} x_{2}, \mathrm{~d} x_{3}, \mathrm{~d} x_{4}\right\}
$$

is spanned not only by $\mathrm{d} x_{1}, \mathrm{~d} \dot{x}_{1}=\mathrm{d} x_{2}, \mathrm{~d} \ddot{x}_{1}=$ $2 x_{3} /\left(A_{g} m \mu_{0}\right) \mathrm{d} x_{3}-2 x_{4} /\left(A_{g} m \mu_{0}\right) \mathrm{d} x_{4}$, but also by $\omega$, where $\omega$ can be chosen either as $\omega=\mathrm{d} x_{3}$ or $\omega=\mathrm{d} x_{4}$ to get the complete basis of $\mathcal{H}_{1}$. This also means that $\mathrm{d} x_{3}$ (or $\mathrm{d} x_{4}$ ) is the starting element of the second Brunovsky chain. The flat outputs are given by the starting elements of Brunovsky chains. Therefore, we obtain $\tilde{y}_{1}=x_{1}$, but for the second flat output we have two options: either $\tilde{y}_{2}=x_{3}$ or $\tilde{y}_{2}=x_{4}$. Recall that both $x_{3}$ and $x_{4}$ are computable from (16b).

Next, we apply the i/o linearization procedure from Section 2 to flat outputs. Let us first consider the case when $\tilde{y}_{1}=h_{1}(x)=x_{1}, \tilde{y}_{2}=h_{2}(x)=x_{3}$. The relative degrees $r_{1}=3, r_{2}=1$, the decoupling matrix

$$
\begin{aligned}
\mathbf{A}(x) & =\left[\begin{array}{cc}
L_{g_{1}} L_{f}^{2} h_{1} & L_{g_{2}} L_{f}^{2} h_{1} \\
L_{g_{1}} h_{2} & L_{g_{2}} h_{2}
\end{array}\right] \\
& =\left[\begin{array}{cc}
\frac{2 x_{3}}{A_{g} m N \mu_{0}} & -\frac{2 x_{4}}{A_{g} m N \mu_{0}} \\
\frac{1}{N} & 0
\end{array}\right]
\end{aligned}
$$


and its inverse

$$
\mathbf{A}^{-1}(x)=\left[\begin{array}{cc}
0 & N \\
-\frac{A_{g} m N \mu_{0}}{2 x_{4}} & \frac{N x_{3}}{x_{4}}
\end{array}\right] .
$$

To compute the control, one has, by (6), to compute

$$
\begin{aligned}
& L_{f}^{3} h_{1}=4 R \frac{\left(-s_{0}+x_{1}\right) x_{3}^{2}+\left(s_{0}+x_{1}\right) x_{4}^{2}}{A_{g}^{2} m N^{2} \mu_{0}^{2}}, \\
& L_{f} h_{2}=2 R \frac{\left(-s_{0}+x_{1}\right) x_{3}}{A_{g} N^{2} \mu_{0}}
\end{aligned}
$$

to obtain

$$
\begin{aligned}
& u_{1}=\frac{2 R\left(s_{0}-x_{1}\right) x_{3}}{A_{g} N \mu_{0}}+N v_{2}, \\
& u_{2}=\frac{2 R\left(s_{0}+x_{1}\right) x_{4}}{A_{g} N \mu_{0}}-\frac{N A_{g} m \mu_{0}}{2 x_{4}} v_{1}+\frac{N x_{3}}{x_{4}} v_{2} .
\end{aligned}
$$

Note that application of (32) requires $x_{4} \neq 0$. If $x_{4}=0$, then $u_{2}$ is not determined. However, the case $x_{4}=0$ (or small) can be handled with the different choice of flat outputs, yielding a feedback which is valid for the case $x_{4}=0$ but not valid when $x_{3}=0$ (or small).

The alternative choice of flat outputs $\tilde{y}_{1}=h_{1}(x)=$ $x_{1}, \tilde{y}_{2}=h_{2}(x)=x_{4}$ yields the same relative degrees $r_{1}=3, r_{2}=1$ with a slightly modified decoupling matrix,

$$
\mathbf{A}(x)=\left[\begin{array}{cc}
\frac{2 x_{3}}{A_{g} m N \mu_{0}} & -\frac{2 x_{4}}{A_{g} m N \mu_{0}} \\
0 & \frac{1}{N}
\end{array}\right],
$$

and its inverse,

$$
\mathbf{A}^{-1}(x)=\left[\begin{array}{cc}
\frac{A_{g} m N \mu_{0}}{2 x_{3}} & \frac{N x_{4}}{x_{3}} \\
0 & N
\end{array}\right] .
$$

Next we find

$$
\begin{aligned}
& L_{f}^{3} h_{1}=4 R \frac{s_{0}\left(-x_{3}^{2}+x_{4}^{2}\right)+x_{1}\left(x_{3}^{2}+x_{4}^{2}\right)}{A_{g}^{2} m N^{2} \mu_{0}^{2}}, \\
& L_{f} h_{2}=-2 R \frac{\left(s_{0}+x_{1}\right) x_{4}}{A_{g} N^{2} \mu_{0}}
\end{aligned}
$$

\begin{tabular}{|c|c|c|}
\hline Symbol & Value & Meaning \\
\hline$m[\mathrm{~kg}]$ & 2.5 & mass of the rotor \\
\hline$N$ & 108 & $\begin{array}{l}\text { number of turns of the coil } \\
\text { of each electromagnet }\end{array}$ \\
\hline$R[\Omega]$ & 0.5 & coil resistance \\
\hline$A_{g}\left[\mathrm{~m}^{2}\right]$ & 0.0014 & $\begin{array}{l}\text { cross sectional area } \\
\text { of the electromagnet }\end{array}$ \\
\hline$\mu_{0}[\mathrm{H} / \mathrm{m}]$ & $1.25 \times 10^{-6}$ & permeability of free space \\
\hline$s_{0}[\mathrm{~m}]$ & 0.00058 & length of the air gap \\
\hline$\overline{\left[x_{1}(0)\right]}$ & {$[250]$} & \\
\hline$x_{2}(0)$ & $\times 10^{-6}$ & initial values for \\
\hline$x_{3}(0)$ & $\times 10$ & system states \\
\hline$\left\lfloor x_{4}(0)\right.$ & {$[1]$} & \\
\hline$f_{e}[\mathrm{~N}]$ & 0.1 & $\begin{array}{l}\text { maximal value of the load } \\
\text { force disturbance }\end{array}$ \\
\hline$\epsilon$ & $1 \times 10^{-6}$ & $\begin{array}{l}\text { boundary of the } \\
\text { electromagnetic flux } \\
\text { near the origin }\end{array}$ \\
\hline$k$ & 1000 & feedback gain \\
\hline$\left[\alpha^{0}\right]$ & {$[0.2]$} & \\
\hline$\alpha^{1}$ & 8 & constant gains of $v$ \\
\hline$\alpha^{2}$ & [180 & \\
\hline$\alpha_{1}^{0}$ & {$[9000]$} & \\
\hline$\alpha_{1}^{1}$ & 24000 & $\begin{array}{l}\text { constant gains of } v_{1} \\
\text { for the controllers } 32,\end{array}$ \\
\hline & 2200 & constant gain of $v_{0}$ \\
\hline$\alpha_{2}^{0}$ & 0.1 & for the controllers 32,36 \\
\hline
\end{tabular}

and controls

$$
\begin{aligned}
& u_{1}=\frac{2 R\left(s_{0}-x_{1}\right) x_{3}}{A_{g} N \mu_{0}}+\frac{N A_{g} m \mu_{0}}{2 x_{3}} v_{1}+\frac{N x_{4}}{x_{3}} v_{2} \\
& u_{2}=\frac{2 R\left(s_{0}+x_{1}\right) x_{4}}{A_{g} N \mu_{0}}+N v_{2} .
\end{aligned}
$$

If $x_{3}=0$, then $u_{1}$ is not determined. Since $x_{3}$ and $x_{4}$ represent the components of the magnetic flux in a stable flux-controlled AMB system, either $x_{3} \neq 0$ or $x_{4} \neq 0$. To
Table 1. Parameters of simulation and AMB systems.

avoid singularities, the control law can be defined by (32) if $x_{4} \neq 0$ and by (36) if $x_{3} \neq 0$. If $x_{3}$ and $x_{4}$ are both nonzero, then the choice is arbitrary.

We specify $v_{1}$ and $v_{2}$ for (32) and (36). Recall that for both the choices of flat outputs $r_{1}=3, r_{2}=1$. Let the desired trajectory $\tilde{y}_{d}^{1}=0$ and $\tilde{y}_{d}^{2}=0$. Then from (7) we obtain

$$
\begin{aligned}
& v_{1}=-\left(\alpha_{1}^{2} \ddot{\tilde{y}}_{1}+\alpha_{1}^{1} \dot{\tilde{y}}_{1}+\alpha_{1}^{0} \tilde{y}_{1}\right), \\
& v_{2}=-\left(\alpha_{2}^{0} \tilde{y}_{2}\right) .
\end{aligned}
$$

\section{Simulation results}

This section presents simulation results both for the third- and fourth-order AMB systems with the feedback linearization controllers (13) and (32), (36), respectively. Simulations are carried out to verify the stability of the nonlinear AMB systems under feedback linearizing control. The main parameters of the system specification and those used in simulations are collected in Table 1. The first two blocks of Table 1 contain physical parameters of the AMB system and initial conditions for the simulations, respectively. The third block represents the parameters of the controllers, whose values were chosen in such way that the overshoot and settling time would be minimal. 
The nonlinear AMB models and feedback linearizing controllers are simulated using the Matlab/Simulink software. In particular, the simulation results present the state responses to initial conditions and disturbance $f_{e}$, given in Table 1

The interconnection of the AMB system with feedback linearization control is shown in Fig. 2. The inner nonlinear feedback loop cancels the nonlinearities. The outer linear feedback loop consist of the linear controller $v$ with constant gains given in Table 1

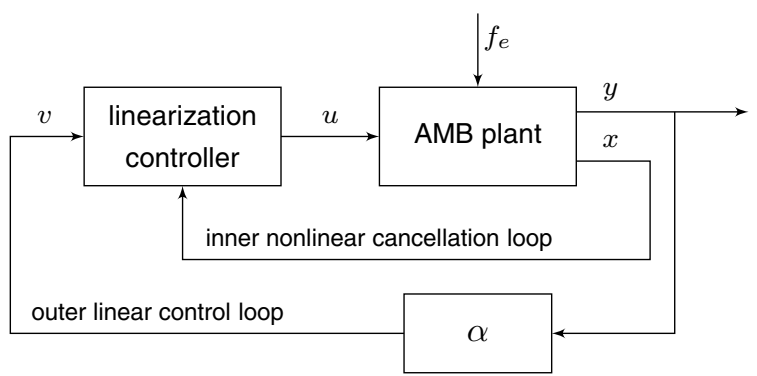

Fig. 2. Interconnections of feedback linearization of the AMB control system.
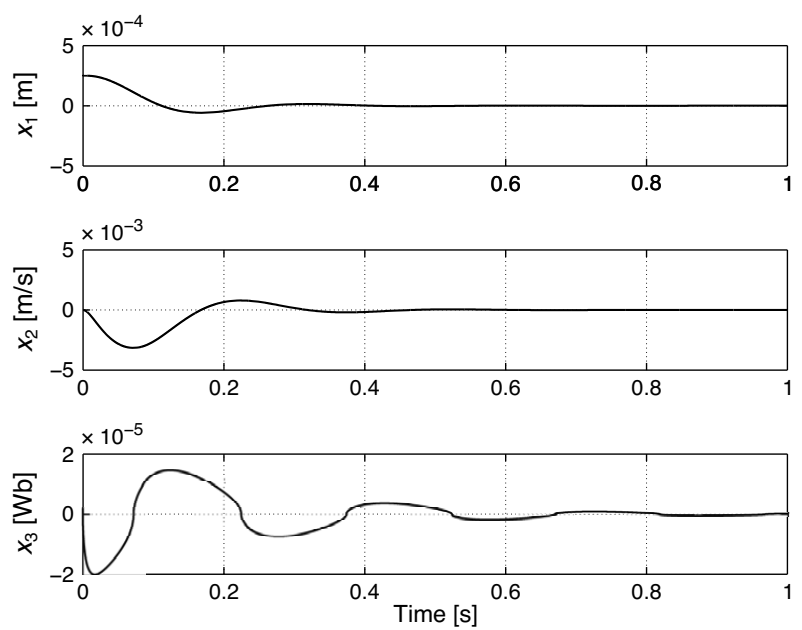

Fig. 3. Responses of the system states to initial conditions.

In simulations only saturations of the controller output were taken into account. Since the dynamics of the mechanical AMB system have some rotor inertia, in simulations the effect of saturation cannot be observed.

4.1. Third-order system. The simulations are performed for the AMB system with zero-bias operation in (8), and using the control voltage switching strategy (9). For a detailed discussion on the AMB system with a control switching scheme, we refer the reader to the works of Mystkowski et al. (2015a; 2015b). Figure 3 presents the responses of the state variables to the initial
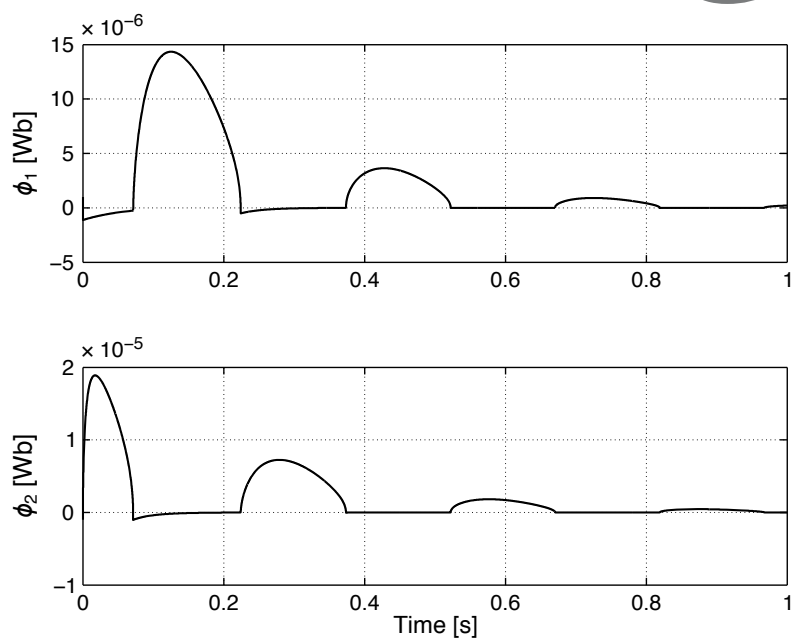

Fig. 4. Responses of electromagnetic fluxes to initial conditions due to control voltage switching operation.
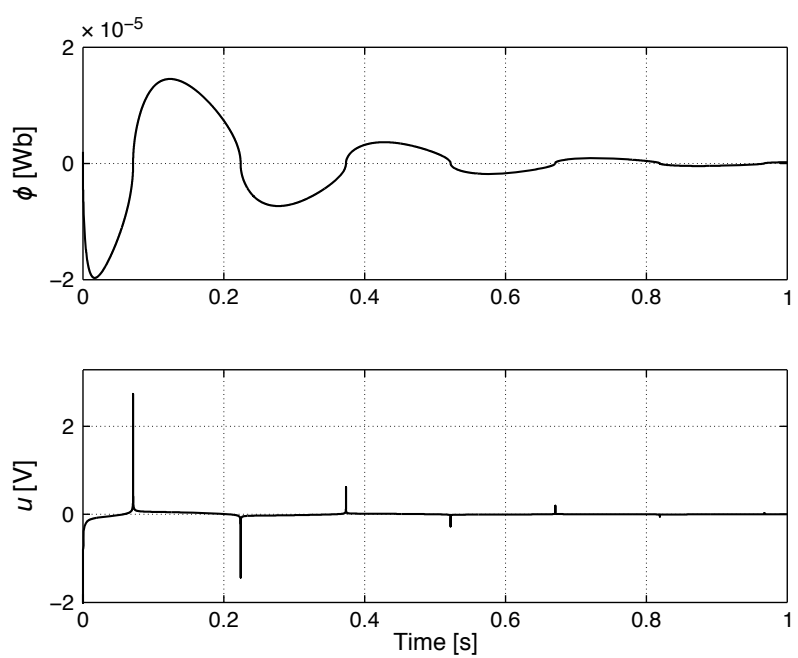

Fig. 5. Responses of the generalized electromagnetic flux and voltage control to initial conditions.

conditions, given in Table 1, when the control voltage switching strategy was applied.

The voltage switching strategy allows controlling both electromagnets, 1 and 2, when only one of them is active at a time. The main reason for this kind of operation is reduction in power loss. The control voltages produce the electromagnetic fluxes, with only one of them being non-zero at any time (see Fig. 4).

Moreover, the controller output $u$ in (13) switches to $-k \operatorname{sgn}\left(x_{3}\right)$ when the boundary $\epsilon$ of the magnetic flux is reached-this guarantees $u$ to be finite even when the state $x_{3}$ is close to zero. The situation can be observed in Fig. 5: the voltage peaks correspond to points, where $x_{3}$ (electromagnetic flux) is crossing zero. Note that the value of $\epsilon$ was chosen to be optimal with respect to both 

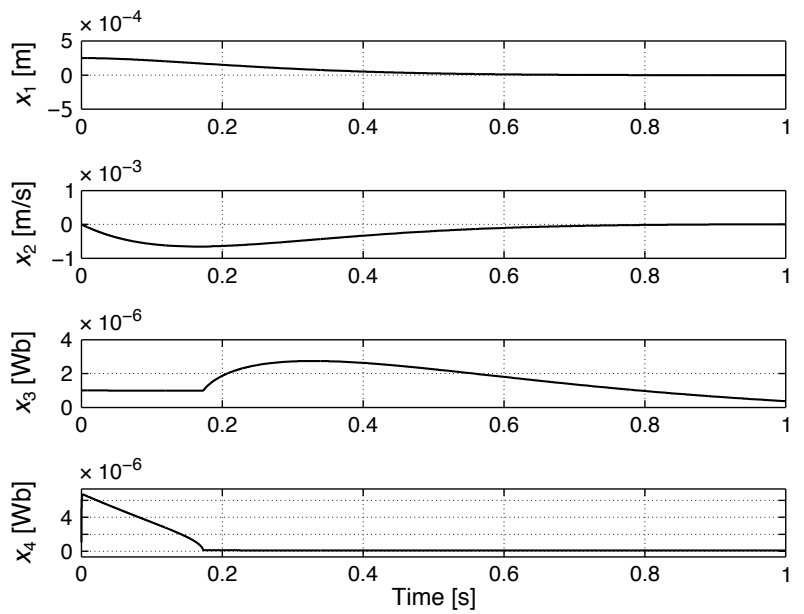

Fig. 6. Responses of the system states to initial conditions.

overshoot and settling time. Additional simulations (not included in the paper) showed that larger $\epsilon$ yields larger overshoot and longer settling time of the AMB system. Furthermore, for substantially larger $\epsilon$, the AMB system state responses become unstable.

4.2. Fourth-order system. The behavior of the fourth-order system under the controllers (32), (36) is investigated numerically. In particular, we apply the controller (32) when $x_{3}$ approaches zero, and the controller (36) when $x_{4}$ is close to zero.

The simulation results show responses of the nonlinear AMB system in a feedback linearization control loop to the initial conditions and load disturbance $f_{e}$.

Figure 6 presents the responses of the state variables. Observe that the controllers (32), (36) work properly and stabilize the system. The switching from the controller (32) to (36) and in the opposite direction does not have a significant effect on the AMB state responses. One can observe that when $x_{3}$ (or $x_{4}$ ) crosses zero, due to the system dynamics, the control current overshoot is negligible and the AMB state responses $x_{1}$ and $x_{2}$ are stable.

In order to verify the robustness of the AMB system, the input load disturbance $f_{e}$ is designed as the square pulse signal acting on the rotor directly. Figure 7 demonstrates the behaviour of the position $x_{1}$ and speed $x_{2}$ of the rotor, when the external load disturbance $f_{e}$ is applied to it. One may observe that stability is ensured at any time when the limited dynamic disturbance is active.

In order to verify the robustness of the feedback linearized AMB system, the additional simulation results of the fourth-order AMB model responses under the high frequency current disturbances are introduced. However, reasonable and realistic simulations can be provided for the current wave, which is generated by two-level
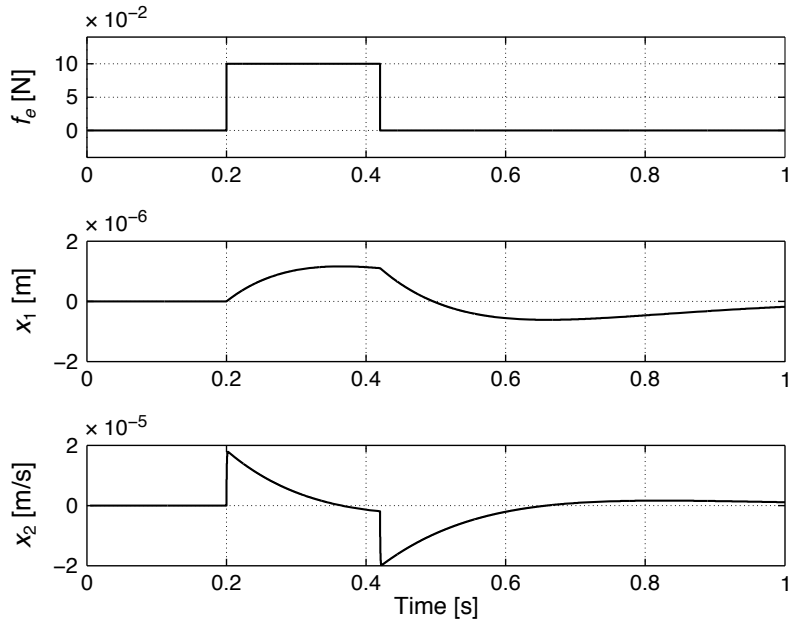

Fig. 7. Responses of the system states to external load disturbance $f_{e}$.
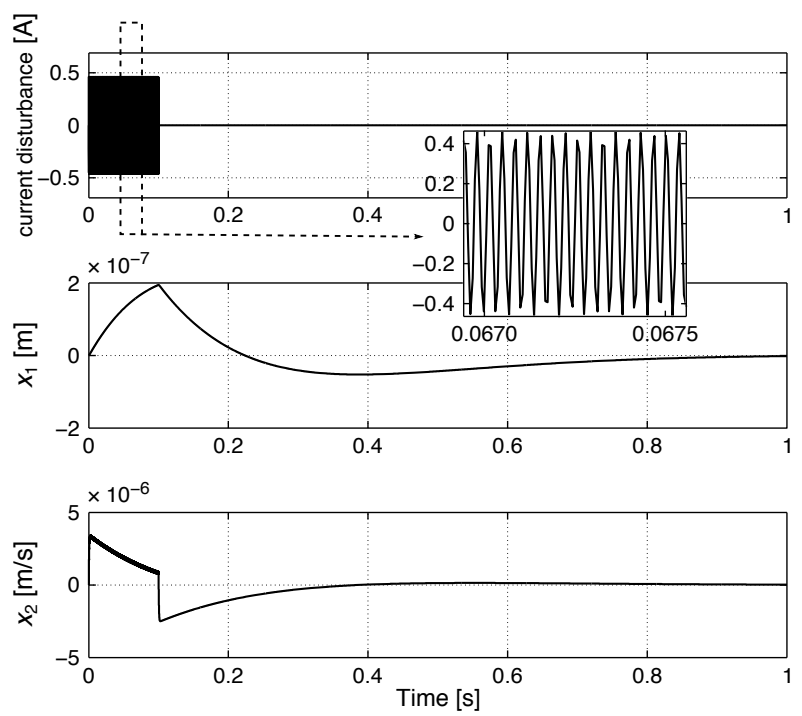

Fig. 8. Responses of the system states to a high frequency sinusoidal disturbance of the control current.

voltage PWM amplifiers operating with an $18 \mathrm{kHz}$ switching frequency. The current ripple depends on the coil inductance which introduces inertia to the system. Therefore, the current ripple amplitude in our AMB system is limited to $0.45 \mathrm{~A}$ with the peak-to-peak value of 0.9 A. A lower current ripple, i.e., in the case of the three-level voltage PWM, would possibly reduce noise in the measured flux signal ripple. Figure 8 presents the rotor displacement and rotor speed responses to the coil current sinusoidal wave disturbances. Disturbance affects the system in the time interval $[0,0.1] \mathrm{s}$. As can be observed, despite the high frequency of the current ripple, the AMB system is stable and the state responses do not show the effect of the disturbance signal. 

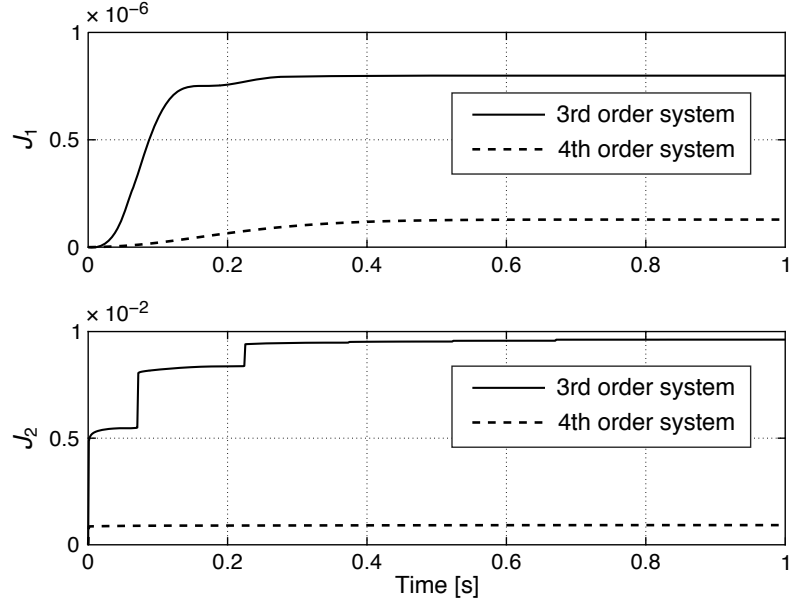

Fig. 9. Comparison of the third- and fourth-order AMB control systems.

In order to assess both the third- and fourth-order AMB control systems, the quadratic cost functions of the states and the inputs (controls)

$$
\begin{aligned}
& J_{1}=\int_{0}^{\infty}\left(x_{1}^{2}+x_{2}^{2}+x_{3}^{2}+x_{4}^{2}\right) \mathrm{d} t, \\
& J_{2}=\int_{0}^{\infty}\left(u_{1}^{2}+u_{2}^{2}\right) \mathrm{d} t,
\end{aligned}
$$

are used. The cost functions (38) for the third- and fourth-order systems are compared and results are shown in Fig. 9.

One may observe that the fourth-order system has a better control performance than the third-order one. Moreover, the total energy required by the controller for the fourth-order system is substantially lower than for the third-order one.

\section{Conclusions}

In the paper the feedback linearizaton technique was effectively applied to the third- and fourth-order AMB systems. The external control loop with a linear controller was used to stabilize the system. Simulation results showed that the closed-loop AMB system is stable with respect to initial conditions and robust with respect to external load disturbances. Based on the simulation results, the controller voltage values are relatively small when the settling time for the rotor position is below $1 \mathrm{~s}$ for all simulations.

The future research on this topic is to verify the suggested approach by means of experiments on the 5-DOF AMB test rig. Moreover, we will investigate how to apply the feedback linearization technique to low-bias AMB systems. Also, we are going to consider a combination of some techniques presented by
Ławryńczuk (2015) and the current approach in order to study predictive control of the AMB system.

\section{Acknowledgment}

This work is supported by the university grant no. S/WM/1/2016 of the Department of Automatic Control and Robotics, Bialystok University of Technology, by the Polish Ministry of Science and Higher Education, and by the Estonian Research Council (personal research funding grant PUT481).

\section{References}

Baloh, M., Tao, G. and Allaire, P. (2000). Modeling and control of a magnetic bearing actuated beam, American Control Conference, Chicago, IL, USA, pp. 1602-1606.

Charara, A., De Miras, J. and Caron, B. (1996). Nonlinear control of a magnetic levitation system without premagnetization, IEEE Transactions on Control Systems Technology 4(5): 513-523.

Chen, M. and Knospe, C.R. (2005). Feedback linearization of active magnetic bearings: Current-mode implementation, IEEE/ASME Transactions on Mechatronics 10(6): 632-639.

Conte, G., Moog, C.H. and Perdon, A.M. (2007). Algebraic Methods for Nonlinear Control Systems. Theory and Applications, 2nd Edn., Springer, London.

Ghosh, J., Mukherjee, D., Baloh, M. and Paden, B. (2000). Nonlinear control of a benchmark beam balance experiment using variable hyperbolic bias, American Control Conference, Chicago, IL, USA, pp. 2149-2153.

Isidori, A. (1995). Nonlinear Control Systems, 3rd Edn., Springer, London.

Jastrzebski, R.P., Smirnov, A., Mystkowski, A. and Pyrhönen, O. (2014). Cascaded position-flux controller for an AMB system operating at zero bias, Energies 7(6): 3561-3575.

Joo, S.J. and Seo, J.H. (1997). Design and analysis of the nonlinear feedback linearizing control for an electromagnetic suspension system, IEEE Transactions on Control Systems Technology 5(1): 135-144.

Ławryńczuk, M. (2015). Nonlinear state-space predictive control with on-line linearisation and state estimation, International Journal of Applied Mathematics and Computer Science 25(4): 833-847, DOI: 10.1515/amcs-2015-0060.

Lévine, J. (2009). Analysis and Control of Nonlinear Systems: A Flatness-Based Approach, Springer, Berlin/Heidelberg.

Lévine, J., Lottin, J. and Ponsart, J.-C. (1996). A nonlinear approach to the control of magnetic bearings, IEEE Transactions on Control Systems Technology 4(5): 524-544.

Lindlau, J.D. and Knospe, C.R. (2002). Feedback linearization of an active magnetic bearing with voltage control, IEEE Transactions on Control Systems Technology 10(1): 21-31. 
Maslen, E.H. (2013). Self-sensing magnetic bearings, in G. Schweitzer and E.H. Maslen (Eds.), Magnetic Bearings: Theory, Design, and Application to Rotating Machinery, Springer, Berlin/Heidelberg, Chapter 15, pp. 435-459.

Matsumura, F., Namerikawa, T. and Murata, N. (1999). Wide area stabilization of a magnetic bearing using exact linearization, Electrical Engineering in Japan 128(2): 53-62.

Mittal, S. and Menq, C.-H. (1997). Precision motion control of a magnetic suspension actuator using a robust nonlinear compensation scheme, IEEE/ASME Transactions on Mechatronics 2(4): 268-280.

Mystkowski, A., Pawluszewicz, E. and Dragašius, E. (2015a). Robust nonlinear position-flux zero-bias control for uncertain AMB system, International Journal of Control 88(8): 1619-1629.

Mystkowski, A., Pawluszewicz, E. and Jastrzebski, R.P. (2015b). Nonlinear position-flux zero-bias control for AMB system, 20th International Conference on Methods and Models in Automation and Robotics, Międzyzdroje, Poland, pp. 471-476.

Naz, N., Malik, M.B. and Salman, M. (2013). Real time implementation of feedback linearizing controllers for magnetic levitation system, IEEE Conference on Systems, Process and Control, Kuala Lumpur, Malaysia, pp. 52-56.

Nijmeijer, H. and van der Schaft, A.J. (1990). Nonlinear Dynamical Control Systems, Springer, New York, NY.

Sira-Ramírez, H. and Agrawal, S.K. (2004). Differentially Flat Systems, Automation and Control Engineering, Marcel Dekker, Inc., New York, NY.

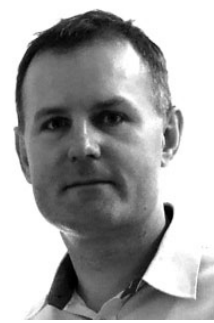

Arkadiusz Mystkowski (b. 1978) received his MSc degree in mechanical engineering with distinction in 2003 from the Bialystok University of Technology. He obtained his $\mathrm{PhD}$ degree in automatic control and robotics with honors in 2007 and his DSc degree in automatic control and robotics in 2016 from the AGH University of Science and Technology in Cracow. From 2003 to 2007 he held the position of an assistant and since 2007 he has been a postdoctoral researcher at the Faculty of Mechanical Engineering, Bialystok University of Technology. He has published over 80 papers in the area of robust optimal control applications: magnetic bearings, dynamics of rotating machinery and unmanned aerial vehicles. His research interests include robust control methods $\left(H_{\infty}, H_{\infty} / H_{2}\right), \mu$-synthesis and loop-shaping, structured and parametric uncertainty design, feedback linearization, control Lyapunov functions, sliding-mode controller and observers.
Vadim Kaparin received the PhD degree in computer and systems engineering from the Tallinn University of Technology in 2013. Currently he is a researcher at the Department of Software Sciences, Tallinn University of Technology. His main research interests lie in the domain of nonlinear control systems theory, both for discrete- and continuous-time cases, as well as for systems on time scales.

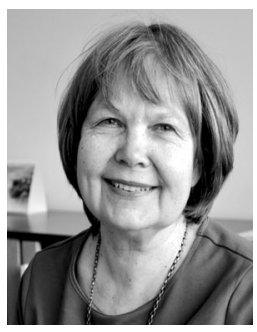

Ülle Kotta received a $\mathrm{PhD}$ degree from the Estonian Academy of Sciences in 1980 and a DSc degree from the Russian Academy of Sciences in 1993. In 1996 she was awarded the prestigious Estonian Science Award. She is the author of the research monograph Inversion Method in the Discrete-time Nonlinear Control Systems Synthesis Problems (Springer, London, 1995) and a (co)author of more than 200 refereed papers. She has supervised numerous PhD students/postdocs and has been a member of the editorial board of three international journals. Between 1997 and 2009 she served as a vice director of the Institute of Cybernetics at the Tallinn University of Technology. She has been a PI of numerous governmental funding projects and ESF grants. Currently she is a leading researcher and the head of the Control Systems Group at the Department of Software Sciences, Tallinn University of Technology. Her present research interests are in nonlinear control theory.

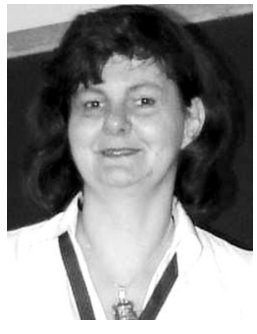

Ewa Pawluszewicz received a $\mathrm{PhD}$ degree in mathematics from the Polish Academy of Sciences in 1998, and habilitation in automatic control and robotics from the AGH University of Science and Technology in 2012. Her interests focus on algebraic and geometric methods in nonlinear control, systems on time scales, and fractional order systems. She is the author and a coauthor of two monographs in Polish, and about 200 papers and book chapters.

Maris Tõnso received a $\mathrm{PhD}$ degree in informatics and systems engineering from the Tallinn University of Technology in 2010. Currently, she is a senior researcher at the Institute of Cybernetics, Tallinn University of Technology. Her research interests focus on the application of polynomial methods to nonlinear control problems. She has been the main developer of the Mathematica package NLControl, developed in the Department of Software Sciences and devoted to modeling, analysis and synthesis problems of nonlinear control systems.

Received: 23 November 2016

Revised: 26 April 2017

Accepted: 31 May 2017 